\title{
Effect of Two Different Superstrate Layers On Bismuth Titanate (BiT) Array Antennas
}

\section{SUBJECT AREAS: \\ ELECTRICAL AND \\ ELECTRONIC \\ ENGINEERING}

ELECTRONIC DEVICES

Received

25 July 2013

Accepted

11 December 2013

Published

15 January 2014

Correspondence and requests for materials should be addressed to

F.H.W.

(weefwenhoon@ gmail.com)

\author{
F. H. Wee' , F. Malek ${ }^{2}$, A. U. Al-Amani ${ }^{3}$ \& Farid Ghani ${ }^{1}$
}

${ }^{1}$ School of Computer and Communication Engineering, Universiti Malaysia Perlis, Perlis, Malaysia, ${ }^{2}$ School of Electrical Systems Engineering, Universiti Malaysia Perlis, Perlis, Malaysia, ${ }^{3}$ School of Materials and Mineral Resources Engineering, Universiti Sains Malaysia, Malaysia.

The microwave industry has shown increasing interest in electronic ceramic material (ECM) due to its advantages, such as light weight, low cost, low loss, and high dielectric strength. In this paper, simple antennas covered by superstrate layers for $2.30 \mathrm{GHz}$ to $2.50 \mathrm{GHz}$ are proposed. The antennas are compact and have the capability of producing high performance in terms of gain, directivity, and radiation efficiency. Bismuth titanate with high dielectric constant of 21, was utilized as the ECM, while the superstrate layers chosen included a split ring resonator and dielectric material. The superstrate layers were designed for some improvement in the performance of directivity, gain, and return loss. The proposed antennas were simulated and fabricated. The results obtained were small antennas that possess high gain and high directivity with $360^{\circ}$, omni-directional signal transmission that resonant types of conventional dipole antenna cannot achieve. The gain of the antenna with the superstrate layer was enhanced by about $1 \mathrm{dBi}$ over the antenna without a superstrate layer at $2.40 \mathrm{GHz}$.

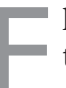
lectronic ceramic material (ECM) has some interesting characteristics, such as small size, ease of fabrication, high radiation efficiency, large impedance width, and low production cost ${ }^{1}$. An ECM antenna can be made much smaller than conventional metal antennas. Quan et al. ${ }^{2}$ worked on broadband, omnidirectional antennas with bandwidths of $800 \mathrm{MHz}$, which was wide enough to cover the frequency bands up to $2.4 \mathrm{GHz}$. The antenna was an array antenna that had $3.8 \mathrm{~cm}$ of electrical size with a gain of $4.5 \mathrm{dBi}$. Hence, this analysis triggered our interest in studying the properties of BiT ECM for potential application on the antenna for the production of miniature, omnidirectional antennas with higher gain, allowing microwave communications that demand the miniaturization of microwave circuits. The characteristics of ECMs make them a better choice for antennas than the conventional, low-gain elements, such as dipoles, monopoles, and microstrip patches ${ }^{3}$. ECM also offers many benefits when used in an antenna because it has no metallic loss and offers high radiation efficiency through millimeter-wave and microwave frequencies ${ }^{4-6}$.

Palukuru et al. ${ }^{7}$ also developed a rectangular-shaped, dielectric resonator antenna. But they used a barium strontium titanate-cylic olefin copolymer (BST-COC) composite dielectric material in an antenna for $2.4 \mathrm{GHz}$ applications. The BST-COC DRA is a very compact antenna that measures $19 \mathrm{~mm} \times 10 \mathrm{~mm} \times 5 \mathrm{~mm}$ and covers 2.4 GHz WLAN applications. However, the research related to the BST-COC antenna was not concern about the performance of the antenna for cases in which the peak gain and radiation efficiency only met the minimum standard gain and radiation efficiency values of a dipole antenna, i.e., $4 \mathrm{dBi}$ and $45 \%$. These poor results occurred due to the low dielectric constant and the high utilization of metal on the antenna due to its copper foil shape and microstrip feed line. The proposed antenna was composed of more than $90 \%$ dielectric material, which covered the transmission line. This can be attributed to the relatively weaker surface currents on the ground plane of DRA in order to achieve high radiation efficiency. Also, an ECM antenna features mechanical simplicity, simple coupling schemes to all commonly-used transmission lines, and ease of excitation. An ECM antenna can use different feed mechanisms, such as probes, microstrip lines, slots, and co-planar lines. An ECM antenna also is more resistant to proximity detuning when it is placed adjacent to, or in the vicinity of, another object.

Microstrip antennas possess major shortcomings, such as narrow impedance bandwidth and low efficiency and gain, which seriously limit their application. Additional research was conducted by Aras et al. ${ }^{8}$ on the dielectric resonator antenna (DRA) using a disk shape. They claimed that the DRA consisted of high dielectric constant materials and was mounted on a dielectric substrate that had lower permittivity. But the authors also claimed that the dielectric material had a high quality factor (Q-factor) that led to a narrower bandwidth of $140 \mathrm{MHz}$ at the resonant frequency of $2.415 \mathrm{GHz}$. The unloaded Q-factor of the proposed antenna was measured at the low Q-factor of 40.2, which is suitable for use in obtaining wider bandwidth. Many broadband techniques 
for microstrip antennas have been reported ${ }^{9,10}$, and to overcome the disadvantage of low gain, some papers has proposed gain enhancement methods using multiple superstrates ${ }^{11,12}$.

The design that was proposed in this study consisted of a rectangular-shaped ECM array antenna with superstrate layers attached. However, the presence of superstrate layers above an antenna may adversely affect the antenna's basic performance characteristics, such as gain, radiation resistance, and efficiency. For this reason, it is important to analyze superstrate effects so adequate superstrate parameters can be chosen to enhance gain and radiation efficiency. It has been reported that higher gain can be achieved if the substrate and superstrate layers are used appropriately ${ }^{11}$. In this study, bismuth titanate $(\mathrm{BiT})$ and a superstrate layer were of considerable interest for a range of applications, including microwave antennas and filters, due to their high dielectric constant and low conduction loss ${ }^{1-6}$. This paper compares the gain of a single patch with superstrate layers with that of a single patch without superstrate layers and reports the experimental results for 6-element, BiT, array antennas with and without a superstrate layer. The details of the proposed antenna design and results are presented in the following sections.

\section{Results}

Electronic ceramic material sample. A bismuth titanate solution was prepared by slowly adding the titanium solution to the prepared bismuth solution to obtain a precursor solution. The precursor solution was stirred continuously and then heated to $80^{\circ} \mathrm{C}$ to form a sticky gel, which was combusted to produce ascombusted powder. The details are described $i^{13}$. Prior to the ECM application, we investigated the crystal structure and surface morphology of the bismuth titanate as $i^{13}$. Based on Scanning Electron Micrograph (SEM), it was apparent that the particles in the bismuth titanate were very dense, free of cracks, and had a few small pores at the grain boundaries and junctions. This agrees with its relative density of about $98 \%$ that had been determined in earlier tests. The X-ray diffraction (XRD) pattern of the sintered BiT observed indicated that very high purity BiT was created and that preferential orientation was obtained ${ }^{14}$. We also investigated the dielectric properties of $\mathrm{BiT}$ in $^{13}$. The tests showed that the $\mathrm{BiT}$ exhibited low losses because it is a free conducting material that has no current flowing on its surface.

Superstrate layers. A split ring resonator (SRR) is one of the elements from metamaterial, which is an artificial media characterized by constitutive parameters generally not found in nature whose values can be engineered to specified values ${ }^{15}$. SRR that exhibits unique properties that do not exist in natural materials has attracted great interest in the last few years ${ }^{15-17}$. SRR is also called left-handed material (LHM), in which the vectors $E, H$, and $k$ form a left-handed system. The SRR exhibited negative permittivity and permeability for which several names and terminologies have been suggested, such as "left handed" media or double-negative (DNG) metamaterials. Since the idea was proposed by Victor Veselago in $1968^{17}$, the availability of such a material has been accepted and extended.

Recently, the demand for planar high directivity antennas, which can be applied to high-speed wireless LAN, satellite reception, and various point-to-point radio links, has increased significantly. In the past few years, new methods for improving the antenna gain have been developed, and the SRR has been proposed ${ }^{18,19}$ and theoretically discussed ${ }^{19}$. In those works, various metallic structures were used as SRR to achieve certain unusual characteristics, which are suitable for high-gain and high-directivity designs.

For a microstrip transmission line of width, w, and a conventional microwave substrate height, $h$, one has the approximate result for the effective relative permittivity of the air-substrate-microstrip system $^{20}$ :

$$
\varepsilon_{e f f}(f)=\varepsilon_{r}-\frac{\varepsilon_{r}-\varepsilon_{e s}}{1+G\left(f / f_{d}\right)^{2}}
$$

where the constants

$$
f_{d}=\frac{Z_{c}}{2 \mu_{0} h} G=0.6+0.0009 Z_{c}
$$

The characteristic impedance is

$$
Z_{c} \cong \frac{1}{2 \pi} \sqrt{\frac{\mu_{0}}{\varepsilon_{e s} \varepsilon_{0}}} \log \left[F_{1} \frac{h}{w}+\sqrt{1+\left(2 \frac{h}{w}\right)^{2}}\right.
$$

With $\left.\mathrm{F}_{1}=6+(2 \pi-6) \exp [-30.666 \mathrm{~h} / \mathrm{w}]^{0.7528}\right]$, and the electrostatic relative permittivity is ${ }^{20}$ :

With

$$
\begin{gathered}
\varepsilon_{e s} \cong \frac{\varepsilon_{r}+1}{2}+\left(\frac{\varepsilon_{r}-1}{2}\right)\left[1+10\left(\frac{h}{w}\right)\right]^{-a b} \\
a=1+\frac{1}{49} \log \left[\frac{\left(\frac{w}{h}\right)^{2}+\left(\frac{w}{52 h}\right)^{2}}{(w / h)^{4}+0.432}\right]+\frac{1}{18.7} \log \left[1+\left(\frac{1}{18.1} \frac{w}{h}\right)^{3}\right. \\
b=0.564\left(\frac{\varepsilon_{r}-0.9}{\varepsilon_{r}+3.0}\right)^{0.053}
\end{gathered}
$$

The goal is to design a length of SRR-loaded transmission line that can be included in some manner with the same length of microstripline to make the paired system dispersionless; that is, we want to produce a dispersion-compensated segment of transmission line. This means we want to introduce a metamaterial with relative permittivity $\varepsilon_{\mathrm{MTM}}$ and permeability $\mu_{\mathrm{MTM}}$ so that the overall relative permittivity and permeability of the system are ${ }^{20}$ :

$$
\frac{\varepsilon(f)}{\varepsilon_{0}}=\varepsilon_{\text {eff }}(f)+\varepsilon_{M T M}(f) ; \frac{\mu(f)}{\mu_{0}}=1+\mu_{M T M}(f)
$$

in such a manner that the wave impedance in the metamaterial remains the same as it is in the original substrate, i.e. ${ }^{20}$ :

$$
Z=\sqrt{\frac{\mu(f)}{\varepsilon(f)}}=Z_{0} \sqrt{\frac{1+\mu_{M T M}(f)}{\varepsilon_{M T M}(f)+\varepsilon_{e f f}(f)}}=Z_{0} \sqrt{\frac{1}{\varepsilon_{e f f}(f)}}
$$

And the index of refraction in the medium compensates for the dispersion effects associated with the microstrip geometry itself; that is the effective index of the pair becomes that of free space ${ }^{20}$ :

$$
\begin{aligned}
n_{e f f}(f) & =\sqrt{\varepsilon_{e f f}(f)}+\sqrt{\frac{\varepsilon(f)}{\varepsilon_{0}}} \sqrt{\frac{\mu(f)}{\mu_{0}}} \\
& =\sqrt{\varepsilon_{e f f}(f)}+\sqrt{\varepsilon_{e f f}(f)+\varepsilon_{M T M}(f)} \sqrt{1+\mu_{M T M}(f)}=1
\end{aligned}
$$

These conditions are satisfied if

$$
\varepsilon_{e f f}(f)\left[1+\mu_{M T M}(f)\right]=\varepsilon_{M T M}(f)+\varepsilon_{e f f}(f)
$$

So that

$$
\mu_{M T M}(f)=\frac{1}{\sqrt{\varepsilon_{e f f}(f)}}-1 \varepsilon_{M T M}(f)=\varepsilon_{e f f}(f) \mu_{M T M}(f)
$$

A high gain and high directive microstrip patch antenna formed with bismuth titanate (BiT) material and covered with Hexagonal Split Ring Resonator (HSRR) substrate was investigated. High-gain. lowprofile antenna design using SRR technology operating at WiMAX and WLAN band (2.30-2.50 GHz) applications was designed and simulated. The Computer Simulation Technology (CST) Microwave Studio was used for the simulation. The dimensions of the split ring 
structure are shown in Fig. 1(a). Nine unit cells of SRR with the same hexagonal structure were combined into a slab to develop a split ring resonator cover. The constructed slab is shown in Fig. 1(b). The constructed slab was located at a distance, $d$, from the conventional antenna to act as a cover. The distance, $\mathrm{d}$, can be varied to obtain the best return loss and radiation pattern. The combination of the SRR slab and the BiT array antenna will be further discussed in ECM Array Antenna with Superstrate Layers section.

Another method was chosen in order to obtain high gain and directivity of this antenna, i.e., a dielectric layer with appropriate thickness and loss tangent. The thin dielectric layer that helps to reduce weight also was able to reduce the surface wave losses. The dielectric layer's dielectric constant, $\varepsilon^{\prime}$, has an important role similar to that of the thickness of the dielectric layer. A low value of $\varepsilon^{\prime}$ for the dielectric layer will increase the fringing field of the patch and thus increase the radiated power ${ }^{21}$. A large gain can be obtained for radiation at any desired angle in the form of a pencil beam at broadside or a conical beam pointing at an angle, $\theta^{22}$. The dielectric layer will act as an optimum reflecting surface when the thickness is chosen so that it is an odd multiple of one quarter of a wavelength in the vertical direction. This corresponds to the condition ${ }^{22}$ :

$$
t=\frac{(2 m-1) \lambda}{4 \sqrt{n_{2}^{2}-\sin ^{2} \theta}}
$$

where $m$ is a positive integer, and $n_{2}$ is the refractive index of the dielectric layer. Using $m=1$, gives the thinnest dielectric layer.

The resonant frequency results from the relationship between the speed of light in a material and the dielectric permittivity are shown in equation $12^{23}$ :

$$
c=\frac{1}{\sqrt{\mu}}=\frac{c_{0}}{\sqrt{r \mu_{r}}}
$$

Thus, as the relative permittivity is increased, the speed of light in a material decreases. For a resonant structure, this slower speed means an object loaded with the dielectric materials of $\varepsilon_{\mathrm{r}}>1$ will have a lower resonant frequency than an unloaded, identical-size structure. Therefore, these loaded structures are said to be "electrically larger" than their unloaded counterparts of the same physical size.

The resonant frequency of the dielectric layer can be calculated from equations (13)-(16) ${ }^{23}$. Equation (13) represents an effective relative permittivity $\varepsilon_{\text {reff }}$, which is a modified relative permittivity value that accounts for the fields fringing in the air above the substrate material ${ }^{23}$.

$$
\varepsilon_{\text {reff }}=\frac{\varepsilon_{r}+1}{2}+\frac{\varepsilon_{r}-1}{2} \frac{1}{\sqrt{1+12 \frac{h}{w}}}
$$

Then, this modified relative permittivity value is used to find the length extension $\Delta \mathrm{L}$ that accounts for the fringing fields at the each of the radiating edges ${ }^{23}$.

$$
\Delta L=h 0.412 \frac{\left(\varepsilon_{\text {reff }}+0.3\right)\left(\frac{W}{h}+0.264\right)}{\left(\varepsilon_{\text {reff }}-0.258\right)\left(\frac{W}{h}+0.8\right)}
$$

The effective length $\mathrm{L}_{\text {eff }}$, can be calculated using the results of equation $(15)^{23}$ :

$$
L_{e f f}=L+2 \Delta L
$$

This allows the resonant frequency to be calculated using the new effective length, as shown in equation $(16)^{23}$ :

$$
f_{r}=\frac{c_{0}}{2\left(L_{e f f}\right) \sqrt{\varepsilon_{r e f f}}}
$$

The resonant frequency and the permittivity are inversely related, such that increasing the permittivity decreases the resonant frequency of the antenna. This allows the production of a simple, significantly miniaturized antenna without adding complexity to the metal patch, such as a slot or a slit for the wideband antenna or the large array patches used by larger antennas for higher gain. Since a simple ECM can be etched onto a high permittivity substrate to achieve a smaller size for a given operating frequency without modifying its shape, this can be beneficial for manufacturing and for mechanical robustness.

A low-loss tangent reduces the dielectric loss and therefore increases the efficiency of the antenna ${ }^{22}$. Thus, the parameters of the dielectric layer so chosen are Tarconic CER-10, with a thickness of $0.5 \mathrm{~mm}$, permittivity of $\varepsilon^{\prime}=10$, and a loss tangent, $\tan \delta=$ $0.0004^{22}$. The optimum sizes obtained were $20 \times 25 \times 0.5 \mathrm{~mm}$, as shown in Fig. 2, with the length of the dielectric layer being shorter

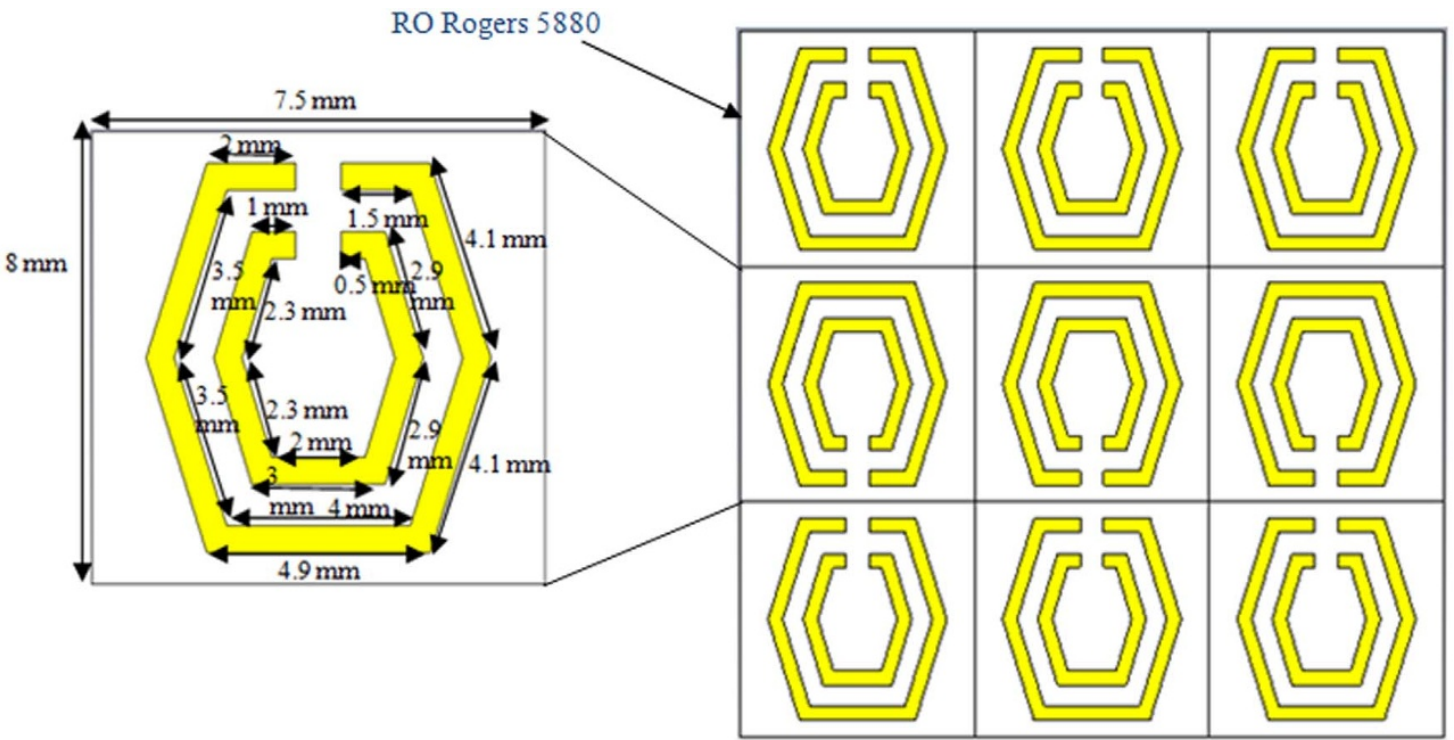

Figure 1 | SRR (a) Dimensions of the single unit cell construction using split ring structures; (b) A SRR slab from a combination of nine unit cells. The HSRR layer consists of $3 \times 3$ units, so the size of the substrate and the cover is $22.5 \mathrm{~mm} \times 24 \mathrm{~mm}$. The width and the split distances of HSRR are $0.6 \mathrm{~mm}$ and $1 \mathrm{~mm}$, respectively. 


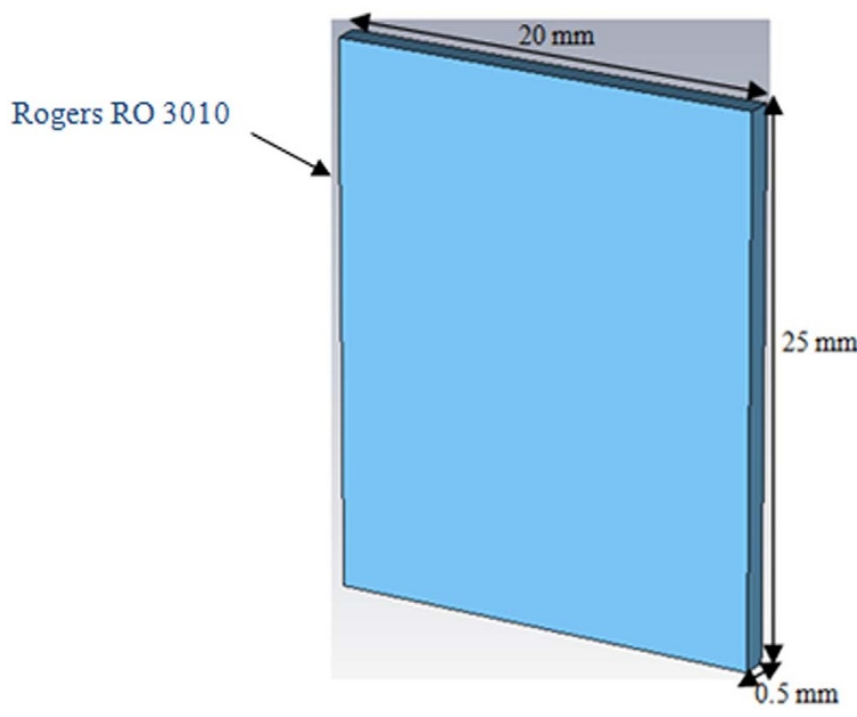

Figure $2 \mid$ Dimensions of the dielectric layer. Roger substrate board, RO 3010 was used as the dielectric layer that covered the six elements BiT array antenna. It comprises $\varepsilon^{\prime}=10.2$ (dielectric constant) with size of $20 \times 25 \times 0.5 \mathrm{~mm}$.

than the length of the HSRR layer. This condition is to make sure the resonant frequency is $2.4 \mathrm{GHz}$, according to the equation (16). The details on the combination of dielectric layer and BiT array antenna will be discussed in ECM Array Antenna with Superstrate Layers section.

ECM array antenna. The frequency behavior of the ECM antenna suggests that the antenna supports both the WLAN and WiMAX ranges. In designing an ECM array antenna, one also must consider the possibility of the high loss associated with the antenna. Numerical simulation shows high levels of conduction loss and power loss with the utilization a metallic structure for an antenna. Therefore, ECM array antennas were designed with low loss BiT elements that present negligible metallic loss in the structure of the antenna, thereby reducing the loss factor that will lead to low performance of the antenna ${ }^{13}$. One of the principle shortcomings is that a microstrip array antenna with a feed network exhibits a relatively large insertion loss, especially when the array is high in surface current or when it is operating at higher frequencies. At microwave frequencies, the loss in copper transmission lines becomes significant. Thus, it is crucially important to minimize insertion loss when designing the power division transmission lines. A series-feed, linear array results in a more compact, lowerloss network that requires less transmission line lengths and fewer junctions that parallel-feed arrays. This results in a lower insertion loss than the parallel feed ${ }^{13}$.

The design for the series-fed, linear, ECM-array antenna was based on a resonant-array approach, in which a standing wave was assumed on the microstrip antenna. This assumption led to a fairly good prediction of the miniaturization of the array when the number of ECM elements is less and the amount of coupling from the microstrip line for each ECM array element is relatively low. For greater numbers of ECM elements, the coupling to each element is greater. Thus, for the proposed ECM array antenna that is designed in array form and has a smaller size, the standing wave also is to be presented in each of the ECMs, leading to high coupling with better performance of the antenna. The layout structure of the six-element, BiT array antenna is shown in Fig. 3. The height, $h$, of the antenna was $25 \mathrm{~mm}$. The antenna was composed basically of four parts, i.e., three branching strips, six radiating patches, microstrip transmission lines, and the feed line. Those four parts were attached to the opposite sides
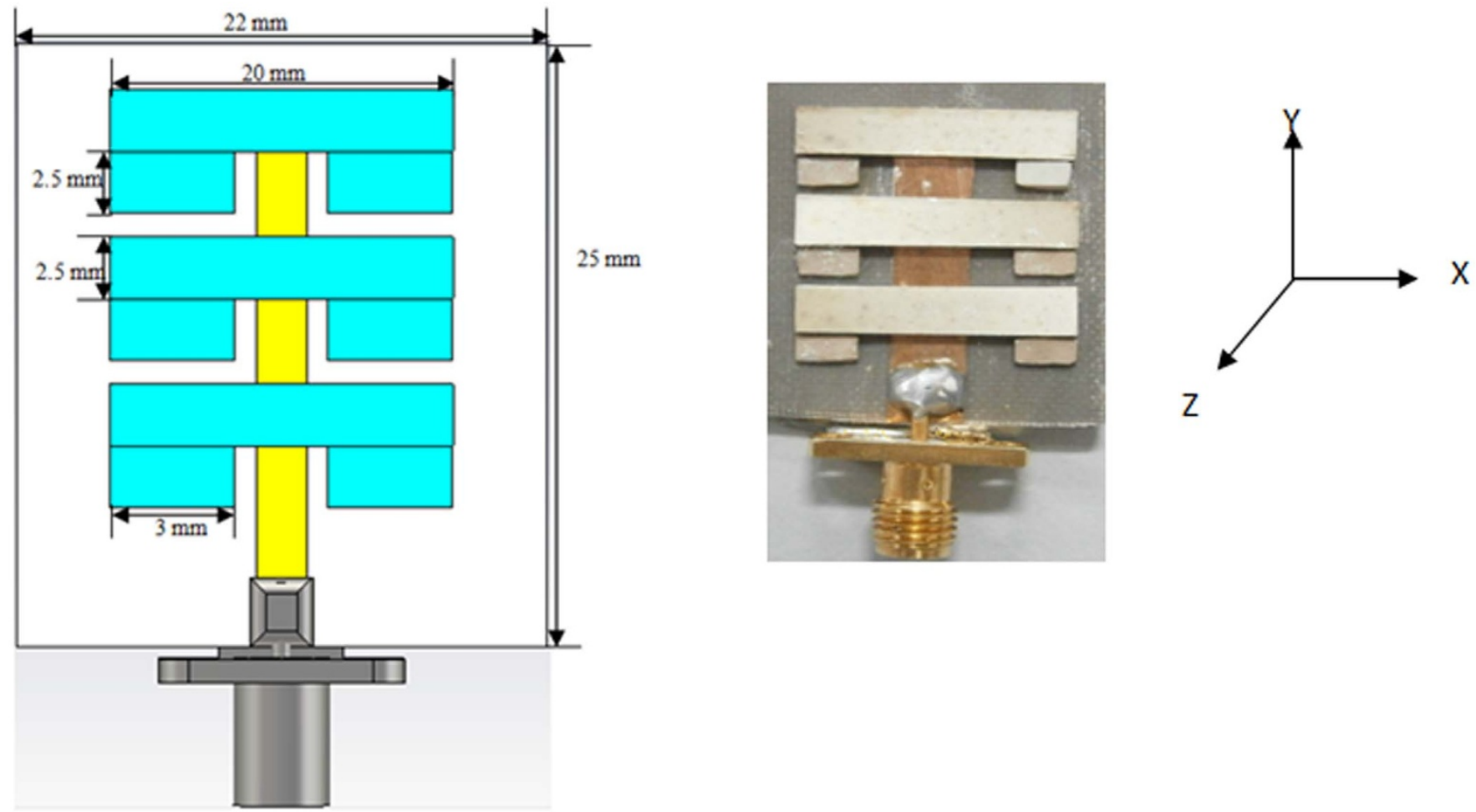

Figure 3 Six elements of the BiT array antenna layout (a) simulated (b) measured. Analysis of the simulation results was conducted to get optimum results before proceeding to the fabrication process. The fabrication process was done on the Roger Corporation's model RT/Duroid 5880 microwave substrate board with a dielectric constant of $\varepsilon^{\prime}=2.2$, a substrate thickness of $\mathrm{h}=1.575 \mathrm{~mm}$, and a loss tangent of tan delta $=0.019$ using the wet-etching technique. 

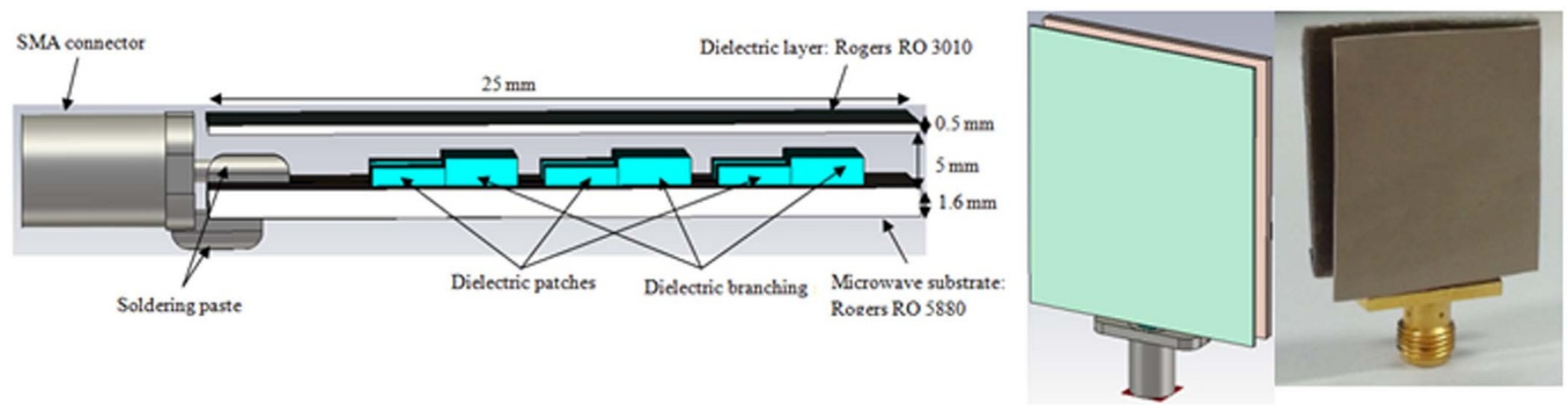

Figure $4 \mid$ Photograph of BiT array antennas with dielectric layer (a) simulated (b) fabricated. The antennas are composed of the radiating element and the dielectric layer with $\varepsilon^{\prime}=10.2$ and thickness $\mathrm{d}=0.5 \mathrm{~mm}\left(0.004 \lambda_{\mathrm{g}}\right.$ at $\left.2.4 \mathrm{GHz}\right)$. The superstrate is placed above the radiating patch at a distance $5 \mathrm{~mm}\left(0.04 \lambda_{0}\right.$ at $\left.2.4 \mathrm{GHz}\right)$, where $\lambda_{0}$ is the wavelength in free space, and $\lambda_{\mathrm{g}}$ is the wavelength in the medium of the superstrate $(\varepsilon=10.2)$.

of a Rogers substrate board, RO Duroid 5880 substrate with dielectric constant, $\mathrm{e}^{\prime}$, of 2.2 and a thickness, $\mathrm{t}$, of $1.6 \mathrm{~mm}$. The parameters of the BiT array antenna were taken from ${ }^{13}$.

ECM array antenna with superstrate layers. Superstrate layers with dimensions of $25 \times 20 \times 0.5 \mathrm{~mm}$ and $24 \times 22.5 \times 1.6 \mathrm{~mm}$ for dielectric layer and SRR layer, respectively, were placed above the radiating patch BiT elements at a distance of $\mathrm{d}=5 \mathrm{~mm}^{22}$ as shown in Fig. 4 and Fig. 5 respectively. The distance of $5 \mathrm{~mm}$ between superstrate layer with BiT antenna patch is the optimum distance at which to obtain high gain and high directivity. In this section, we describe our experimental investigation of the effect of a dielectric layer and SRR layer as the superstrate layer for high gain and high directivity on the six elements of the BiT array antenna, and we present the comparison between BiT array antenna with and without the superstrate layer.

The dielectric layer used was Rogers RO 3010 material due to its ability in reducing the surface losses, thereby leading to high radiation performance. The combination of RO 5880 and the hexagonal structure made of perfect electric conductor (PEC) was used to build up the HSRR substrate. The PEC is very important for assuming the ideal case during the simulation to obtain the best results from the SRR. The detailed features are shown in Table 1.

The performance of the simulated BiT array antennas with the superstrate layers was analyzed using Computer Simulation Technology (CST) Microwave Studio, while the fabricated BiT array antenna with and without the superstrate layers were tested using performance network analyzer (PNA) and near-field test facility that available in university's laboratory.

Fig. 6 shows a graph of return loss with the stacked of the superstrate layers on BiT array antennas. The simulated and measured return losses obtained were functions of the frequency. Here, the frequency bandwidth of the antenna was defined as a return loss less than $10 \mathrm{~dB}$. For the results of BiT array antenna with dielectric layer, the return loss was reduced to a highest value of $24.5 \mathrm{~dB}$ and $27.5 \mathrm{~dB}$ for the simulation and measurement results, respectively, as compared with the antenna without dielectric layer, which was $20 \mathrm{~dB}$ and $23 \mathrm{~dB}$, respectively. This condition showed that the capacitive effects of placing the dielectric over the antenna did not affect the antenna's performance.

From the results of the BiT array antenna with SRR layer, the return loss seems to have a similar reflectivity effect, which both antennas own the best transmission of signal compared to six-element BiT array antenna without superstrate layer. However, these results did not affect the overall performances of the BiT array antennas with and without the superstrate layer because their impedance matching was acceptable with more than $90 \%$ match.

The operating bandwidths of the proposed, six-element, BiT array antennas with and without the superstrate layers were found to be about $600 \mathrm{MHz}$ for the two superstrate layers and $200 \mathrm{MHz}$ without superstrate layer, respectively, which is compatible with the specification of the WLAN and WiMAX systems. Overall, good agreements were found between the simulation and measurement results, although there was a slight discrepancy between them, which was believed to be due to to the environmental effect and mechanical tolerance, which were neglected in our simulations.

Fig. 7 shows the radiation pattern of a six-element, BiT, array antenna and a six-element, $\mathrm{BiT}$, array antenna with superstrate layers in $2 \mathrm{D}$ view, which was analyzed in the vertical plane $(\mathrm{XZ}, \mathrm{YZ})$ and in the horizontal plane (XY) at $2.40 \mathrm{GHz}$. It can be observed that the 2$\mathrm{D}$ radiation pattern of the co-polarization has a null at $\theta=90^{\circ}$. The mode characteristics can be illustrated further by its $3-\mathrm{D}$ patterns.
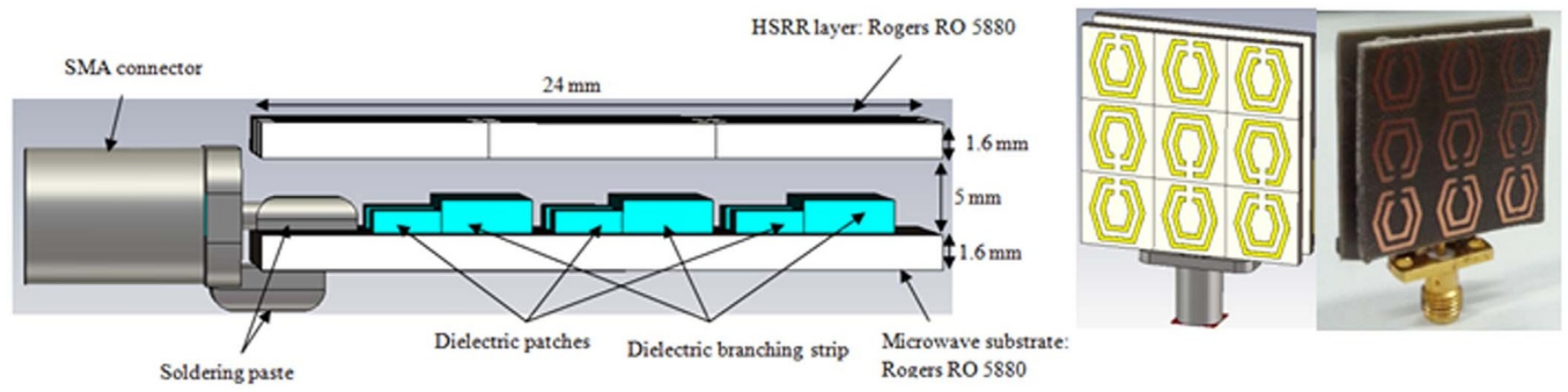

Figure 5 | BiT array antenna with HSRR layer (a) simulated (b) measured. The cover that is made up of a metamaterial layer is an HSRR substrate that is composed of few cells of copper strips with two hexagon shapes in a single $7.5 \mathrm{~mm} \times 8 \mathrm{~mm}$ Rogers (RO 5880) substrate (in one cell). The purpose of the schematic design is for high gain and high directivity on six elements of BiT array antenna. 
Table 1 | Properties of Rogers RO 3010 and Rogers RO 5880 microwave substrate

\begin{tabular}{lcc} 
& \multicolumn{2}{c}{ Values } \\
\cline { 2 - 3 } Properties & Rogers RO 3010 & Rogers RO 5880 \\
\hline Permittivity, $\varepsilon^{\prime}$ & 10.2 & 2.2 \\
Loss Tangent, tan $\delta$ & 0.0004 & 0.025 \\
Permeability, $\mu$ & 1 & 1 \\
Thickness of the substrate, $\dagger$ & $0.5 \mathrm{~mm}$ & $1.6 \mathrm{~mm}$ \\
\hline
\end{tabular}

The beam peak is directional at $\theta=0^{\circ}$, radiating from the center of the patch. Since the ground plane is finite, there is some back radiation that can be characterized by the front-to-back ratio, as mentioned earlier. The pattern resembles a donut shape in which the maximum direction of radiation is at an elevation angle of $\theta=$ $15^{\circ}$ from the z-axis. These antennas provided a 360-degree coverage signal, which is in all directions (horizontally) for two-way communication. The main directivity was directed at the vertical plane, which formed an omni-directional signal level in the H-plane. The H-plane has an important role because this parameter determines the point of strongest signal, thus avoiding signal redundancy and interference with other antennas radiating at the same plane. In this case, along Efield in Fig. 7(a), which correspond to the radiation directly overhead from the antenna, there is little power transmitted from the sixelement, BiT, array antenna as compared to the six-element, BiT, array antenna without superstrate layers. The highest radiation intensity was provided by the six-element, BiT, array antenna with SRR layer. This was because this antenna exhibited the highest gain and directivity, which helped enhance the power signal of the antenna.
From Table 2, the gain of the six-element, BiT, array antenna, in radiation patterns, with dielectric layer and SRR layer exhibited 8.15 and $8.37 \mathrm{~dB}$ of gain and 8.53 and $8.66 \mathrm{dBi}$ of directivity, respectively. The results show that the gain of the superstrate layers stacked on the six-element, BiT, array antenna was improved greatly compared to the common antenna. After studying both antennas, the overall results proved that the $\mathrm{BiT}$ array antenna with superstrate layers had better performances than the antenna that was designed without the superstrate layer.

\section{Discussion}

In the research reported in this paper, we introduced experimentally the effect of a superstrate layer for high gain on a electronic ceramicmaterial antenna. The radiating element with broadband and high radiation efficiency was designed to enhance the narrow bandwidth characteristic by a superstrate with high permittivity. We conducted experiments designed to compare the gain of a six-element, BiT array antenna with a superstrate to that of a six-element, BiT, array antenna without a superstrate layer. The six-element array antenna using superstrate layers had a high gain that exceeded $1 \mathrm{dBi}$ and a wide impedance bandwidth of over $67 \%$. Overall, the six-element, BiT, array antenna with HSRR had the best performance, followed by six-element, BiT, array antenna with dielectric layer, and, finally, the six-element, BiT, array antenna without the superstrate layer. The proposed antenna presented here will be useful for high gain systems that require a broadband bandwidth and will enable them to transmit and receive maximum signals.

\section{Methods}

The six elements of BiT antenna was simulated and fabricated for real application use, the size of the antenna with BiT element loaded was miniaturized. The most popular technique for reducing the size of a printed antenna is the use of a high- dielectric material for the radiating part. In doing so, the guided wavelength underneath the patch can be reduced and hence the size of the resonating patch also can be reduced.

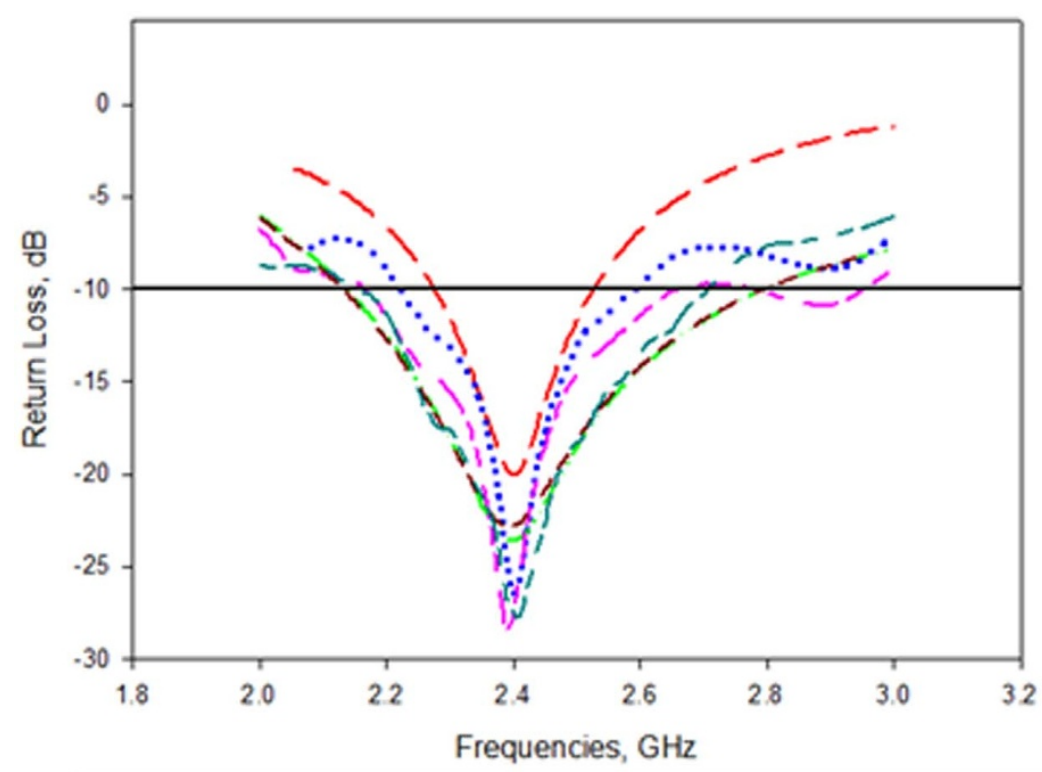

\begin{tabular}{|ll|}
\hline$-\ldots$ & Simulation of BiT array antenna \\
& Measurement of BIT array antenna
\end{tabular}

Figure 6 Comparison of return loss of the simulated and measured BiT array antennas and the BiT array antenna with the superstrate layer. The experimental measurements of the fabricated ECM array antennas with and without superstrate layers were performed using an E8362B performance network analyzer (PNA). 

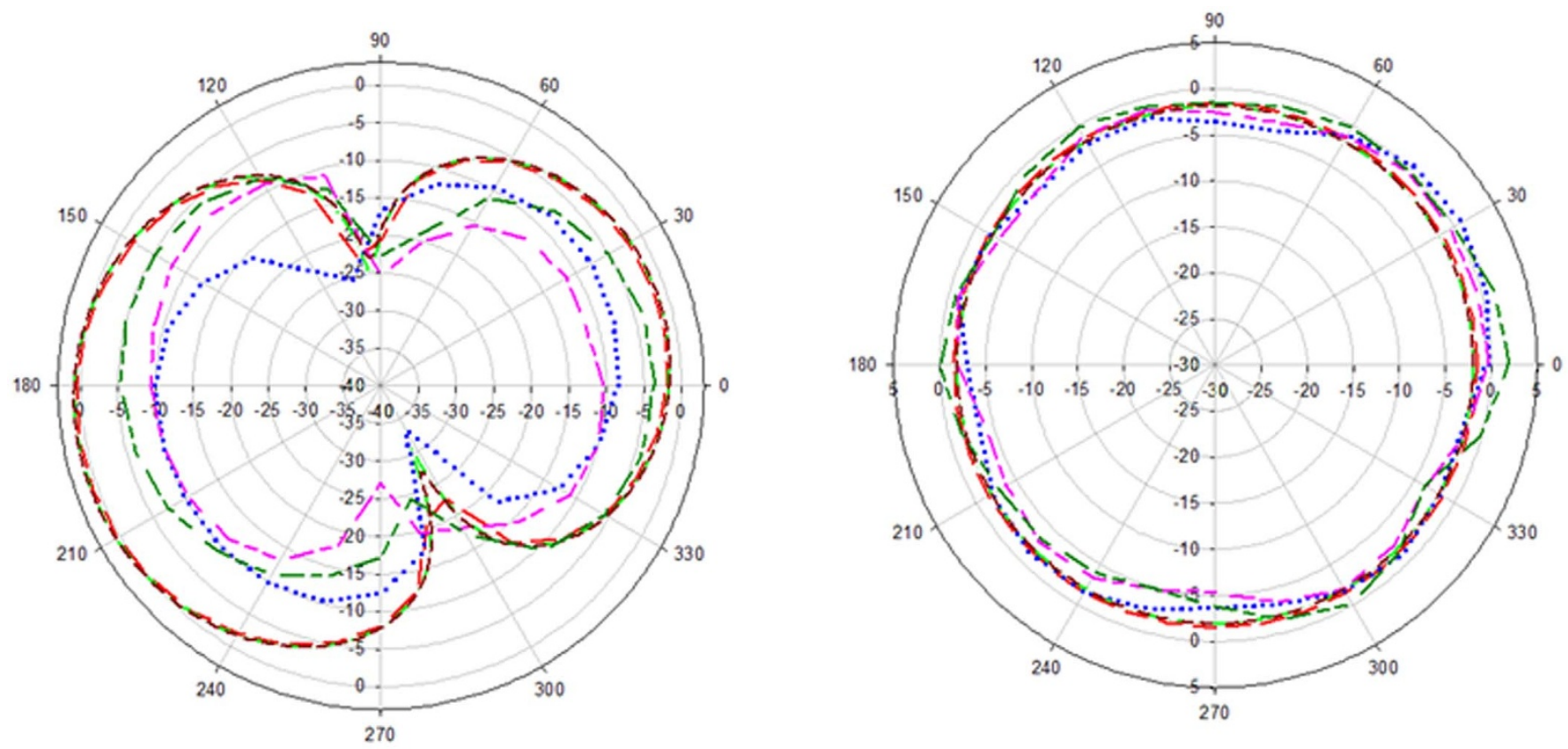

\begin{tabular}{|c|c|}
\hline 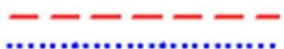 & Simulation of BiT array antenna \\
\hline$-\cdots+\cdots$ & Measurement of BiT array antenna \\
\hline - & Simulation of BT array antenna with dielectric layer \\
\hline$------1--1$ & $\begin{array}{l}\text { Measure ment of } \mathrm{BiT} \text { arfay antenna with die lectric layer } \\
\text { Simulation of BIT array antenna with SRR }\end{array}$ \\
\hline$---\cdots---$ & Measurement of BiT array antenna with SRR \\
\hline
\end{tabular}

Figure $7 \mid$ Radiation pattern in 2-D view (a) E-Field (b) H-Field. Both simulated and measured radiation pattern of the BiT array antenna with and without the dielectric layer are displayed in this figure.

The reduction ratio is approximately related to the square root of $\varepsilon^{\prime 24}$. Existing patch antenna using an $\varepsilon^{\prime \prime}$ values in the range of 1 to 10 have a dimension of about $0.44 \lambda^{25}$ However, for the BiT antenna using an $\mathrm{e}^{\prime}$ value of 21 , the size of the patch is reduced to about $0.20 \lambda$. To further reduce the size, array elements of BiT materials were introduced. Depending on the number of BiT elements added to the patch antenna, a size reduction of about $28 \%$ can be achieved.

\section{Conclusion}

At the present time, antenna miniaturization is of great concern due to the physical benefits, and the design of the dielectric-resonator, ceramic antenna satisfies this need. Even though the proposed design is subject to the same adverse effects as other antennas, i.e., sag in the pattern shape, reduction of hardware measurement bandwidth, and return loss, it is sufficiently compact and has the proper impedance bandwidth for use in the range of $2.3 \mathrm{GHz}$ to $2.5 \mathrm{GHz}$. Ultimately, $\mathrm{BiT}$ array antennas with superstrate layers were tested experimentally to enhance their multiple advantages for use in wireless antenna systems. Compared to available microstrip patch antennas, these advantages include smaller size, higher radiation coefficient properties, wider bandwidths, and high gain and directivity for far distant radiation power. Eventually, our goal relative to BiT array antennas with

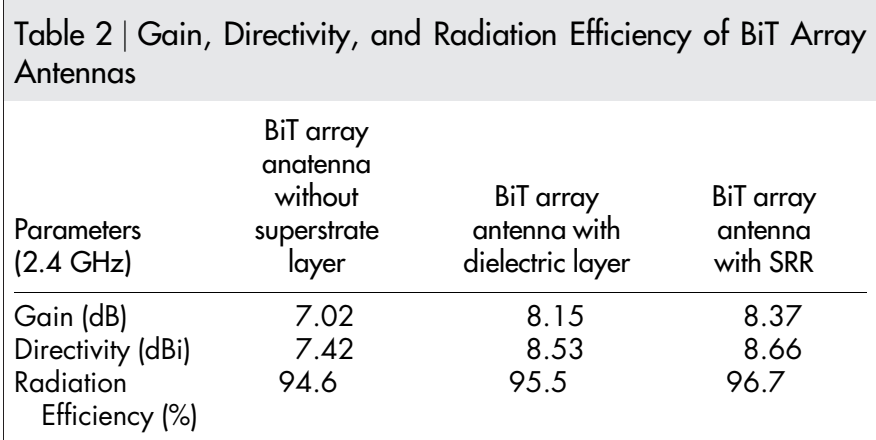

superstrate layers is to provide highly-efficient, miniaturized antennas with large bandwidths, low profiles, and low production costs. Such antennas are certain to be more efficient and effective than the conventional microstrip antennas for modern wireless systems.

1. O'Connor, E. M. \& Long, S. A. The History of the Development of the Dielectric Resonator Antenna. ICEAA International Conference on Electromagnetics in Advanced Applications, Turin, Italy, 872-875, (2007), September 17-21.

2. Quan, X. L., Li, R. L. \& Tentzeris, M. M. A broadband omnidirectional circulary polarized antenna. IEEE Trans. Antennas. and Propag. 61, 2363-2370 (2013).

3. Abdel-Wahab, W. M., Busuioc, D. \& Safavi-Naeini, S. Modeling and design of millimeter wave high $\mathrm{Q}$ factor parallel feeding scheme for dielectric resonator antenna arrays. IEEE Trans. Antennas. and Propag. 59, 2834-2843 (2011).

4. Al-Zoubi, A. S., Kishk, A. A. \& Glisson, A. W. Analysis and design of a rectangular dielectric resonator antenna fed by dielectric image line through narrow slots. Prog Electromagn Res. 77, 379-390 (2007).

5. Ain, M. F. et al. $2.5 \mathrm{GHz} \mathrm{BaTIO}_{3}$ dielectric resonator antenna. Prog Electromagn Res. 76, 201-210 (2007).

6. Long, S. A., McAllister, M. W. \& Shen, L. C. The resonant cylindrical dielectric cavity antenna. IEEE Trans. Antennas. and Propag 31, 406-421 (1983).

7. Palukuru, V. K., Sonoda, K., Surendran, R. \& Jantunen, H. BST COC composite based rectangular dielectric resonator antenna (DRA) for 2.4 WLAN wrist applications. Prog Electromagn Res C. 16, 195-205 (2010).

8. Aras, M. S. M., Rahim, M. K. A., Asrokin, A. \& Aziz, M. Z. A. A. Dielectric resonator antenna (DRA) for wireless application, RF and Microwave Conference RMC2008, 454-458 (2008).

9. Sabban, A. A new broadband stacked two-layer microstrip antenna. IEEE AP-S Int. Symp. Digest. 21, 63-66 (1983).

10. Jang, Y. W. Wide-band t-shaped microstrip-fed twin-slot array antenna. ETRI J. 23, 33-38 (2001).

11. Jackson, D. R. \& Alexopoulos, N. G. Gain enhancement methods for printed circuit antennas. IEEE Trans. Antennas. and Propag, 33, 976-987 (1985).

12. Yang, H. Y. \& Alexopoulos, N. G. Gain enhancement methods for printed circuit antennas through multiple superstrates. IEEE Trans. Antennas. and Propag 35, 860-863 (1987).

13. Wee, F. H., Malek, F., Ghani, F. \& Al-Amani, A. U. Integration of miniaturized array antenna with high-permittivity rectangular bismuth titanate for WiMAX applications. IEEE Trans. Antennas. and Propag. 60, 6032-6036 (2012). 
14. Sreekantan, S., Noor, A. F. M., Ahmad, Z. A., Othman, R. \& West, A. Structural and electrical characteristics of crystalline barium titanate synthesized by low temperature aqueous method. J. Mater. Process. Technol. 195, 171-177 (2008).

15. Smith, D. R., Padilla, W. J., Vier, D. C., Nemat-Nasser, S. C. \& Schultz, S. Composite medium with simultaneously negative permeability and permittivity. Phys. Rev. Lett. 84, 4184-4187 (2000).

16. Shelby, R. A., Smith, D. R. \& Shultz, S. Experimental verification of a negative index of refraction. Science. 292, 77-79 (2001).

17. Veselago, V. G. The electrodynamics of substances with simultaneously negative values of $\varepsilon$ and $\mu$. Soviet Phys. Uspekhi. 10, 509-514 (1968).

18. Chen, L., He, S. \& Shen, L. Finite-size effects of a left-hand material slab on the image quality. Phys. Rev. Lett. 92, 1-4 (2004).

19. Islam, M. T., Shakib, M. N. \& Misran, N. High gain microstrip patch antenna. Eur. J. Sci. Res. 32, 187-193 (2009).

20. Wiley-Interscience. Metamaterials physics and engineering explorations. [Engheta, N. \& Ziolkowski, R. W.] [1-438], (Wiley-IEEE Press, August 2006).

21. Wee, F. H., Malek, F., Ghani, F., Sreekantan, S. \& Al-Amani, A. U. High gain and high directive of antenna arrays utilizing dielectric layer on bismuth titanate (BiT) ceramics. IEEE Trans. Antennas. and Propag 1-8 (2012).

22. Li, B. \& Kwok, W. L. Strip-fed rectangular dielectric resonator antennas with/ without a parasitic patch. IEEE Trans. Antennas. and Propag. 53, 2200-2207 (2011).

23. $2^{\text {nd }}$ edition Antenna Theory Antenna Analysis \& Design. [C.A. Balanis][1-1136], (John Wiley \& Sons, Inc., 1993).

24. Kishk, A. A. Wideband truncated tetrahedron dielectric resonator antenna excited by a coaxial probe. IEEE Trans. Antennas. and Propag. 51, 2913-2917 (2003).

25. Gangwar, R. K., Singh, S. P. \& Kumar, D. A modified fractal rectangular curve dielectric resonator antenna for WiMAX application. Prog Electromagn Res. 12, 37-51 (2010).

\section{Acknowledgments}

The authors acknowledge the support of the School of Computer and Communication Engineering, Universiti Malaysia Perlis, which provided access to its test facilities for this study.

\section{Author contributions}

The analysis of electronic ceramic material array antennas was proposed by F.M. and F.G. F.H.W., F.M. and F.G. designed and fabricated the ECM array antenna structures with and without the superstrate layer. A.U.A. performed the ECM analysis and fabricated samples jointly with F.H.W. Characterization experiments were performed by F.H.W. and A.U.A. with assistance from F.M. and F.G. All co-authors contributed to the analysis and interpretation of results. The manuscript was written by F.H.W. and F.M. in consultation with all other co-authors. F.M. supervised the project.

\section{Additional information}

Competing financial interests: F. H. Wee and F. Malek received the short term research grant from Universiti Malaysia Perlis. The remaining authors declare no competing financial interests.

How to cite this article: Wee, F.H., Malek, F., Al-Amani, A.U. \& Ghani, F. Effect of Two Different Superstrate Layers On Bismuth Titanate (BiT) Array Antennas. Sci. Rep. 4, 3709; DOI:10.1038/srep03709 (2014)

\footnotetext{
cc)(i) $($ This work is licensed under a Creative Commons AttributionNonCommercial-NoDerivs 3.0 Unported license. To view a copy of this license, visit http://creativecommons.org/licenses/by-nc-nd/3.0
} 